Recepción: 22/10/2018

Aceptación: 19/11/2018

Publicación: 20/01/2019

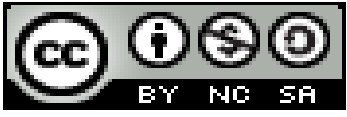

Ciencia de la Educación

Artículo de investigación

\title{
Análisis de conglomerados para la creación de un servicio: una aplicación en la hotelería
}

\section{Conglomerate analysis for the creation of a service: an application in the hotel industry}

\section{Análise de conglomerado para a criação de um serviço: uma aplicação na indústria hoteleira}

\author{
María Claudia Rivera-Prado ${ }^{\mathrm{I}}$ \\ mriveraprado@hotmail.com \\ Arturo Damián Rodríguez-Zambrano II \\ arturo.rodriguez30@gmail.com \\ Sandy Lisbeth Hormaza-Villafuerte III \\ sandy.1isbeth1992@gmail.com
}

Correspondencia: mriveraprado@hotmail.com

\footnotetext{
${ }^{\mathrm{I}}$ Máster Universitario en Marketing e Investigación de Mercados, Licenciada en Ciencias de la Comunicación Mención Publicidad y Mercadotecnia, Docente de la Universidad Laica Eloy Alfaro de Manabí, Manta, Ecuador.

II Magíster en Educación Superior Investigación e Innovaciones Pedagógicas, Ingeniero en Administración de Empresas Turísticas, Docente de Nivelación en la Universidad Laica Eloy Alfaro de Manabí, Manta, Ecuador, Docente de la Universidad Técnica de Manabí, Portoviejo, Ecuador.

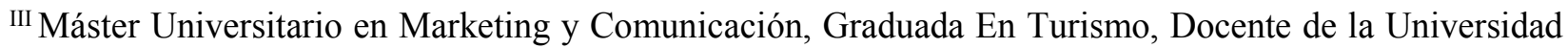
Laica Eloy Alfaro de Manabí, Manta, Ecuador.
} 


\section{Resumen}

El análisis clúster, conocido también como análisis de conglomerados, es una técnica estadística multivariante que busca agrupar elementos o variables tratando de lograr la máxima homogeneidad en cada grupo, lo que aplicado a la hotelería permite diferenciar grupos y establecer un público objetivo a perseguir. El estudio siguió una metodología de tipo descriptiva y bajo un enfoque cuantitativo. Para la recolección de la información se utilizó la técnica de la encuesta online con la plataforma LimeSurvey y el instrumento utilizado se realizó a partir de las indagaciones con los organizadores de bodas. La población estuvo constituida por 162 encuestados que eran especialistas en organización de bodas, de los cuales 103 contestaron todas las preguntas. Entre sus conclusiones se pudo evidenciar que las parejas prácticas se fijan en general en la comida, las parejas emocionales además de la comida y fotografía se preocupan por la luna de miel y en el caso de las parejas exigentes, esperan una alta calidad en todos los aspectos.

Palabras clave: Análisis de conglomerados; hotelería; técnica estadística y especialistas en organización.

\section{Abstract}

The cluster, also known as cluster analysis, analysis is a multivariate statistical technique that seeks to group elements or variables trying to achieve maximum homogeneity in each group, thereby allowing applied to hospitality to differentiate groups and set a target to chase. The study followed a methodology descriptive and under a quantitative approach. The survey technique was used for the collection of information online with the LimeSurvey platform and the instrument used was carried out through inquiries with the organizers of the wedding. The population consisted of 162 respondents who were specialists in organizing weddings, of which 103 answered all the questions. Among their findings demonstrate that practical couples are generally set in the food, emotional couples as well as food and photography are concerned for discerning couples and honeymoon, expect a high quality in all aspects.

Key words: analysis of conglomerates; hospitality; statistical technique and organization specialists. 


\section{Resumo}

A análise de agrupamento, também conhecido como análise de agrupamento é uma técnica estatística multivariada que procura para agrupar elementos ou variáveis tentando alcançar o máximo de homogeneidade em cada grupo, que aplicado à indústria hoteleira diferencia grupos e definir um alvo para prosseguir. O estudo seguiu uma metodologia de tipo descritivo e sob abordagem quantitativa. a técnica de pesquisa online foi usada com a plataforma LimeSurvey para coleta de dados e o instrumento utilizado foi baseado em inquéritos com os planejadores do casamento. A população foi constituída por 162 respondentes especialistas em planejamento de casamentos, dos quais 103 responderam a todas as questões. Entre as suas conclusões poderiam demonstrar que os casais práticas são fixos em geral em alimentos, casais emocionais além de alimentos e fotografia estão preocupados com a lua de mel e, no caso de parceiros que exijam esperam alta qualidade em todos aspectos

Palavras-chave: Análise de conglomerado; hospitalidade; técnica estatística e especialistas em organização

\section{Introducción}

Según diario El Universo (s/f), en Ecuador para realizar una boda convencional, básica y de 150 invitados se necesita un mínimo de 17 mil dólares. Las recepciones suelen celebrarse en salones de eventos y hoteles, por lo que es uno de los ingresos más significativos en este negocio, ya que nutre en una sola noche la categoría de alimentos y bebidas; incluyéndose además la ganancia de otros proveedores para su operación.

El vestido, los zapatos, el velo, los accesorios, las invitaciones, el local, los detalles de la recepción, el fotógrafo y la música son algunos de los gastos que normalmente cubren los novios y sus familias para celebrar su casamiento. En el caso de una boda de destino, en esta se suele utilizar reserva de habitaciones para los novios e invitados y si es en un lugar fuera de la ciudad de origen de los anfitriones, la oportunidad de negocio para los proveedores se expande.

Para la realización de esta propuesta, se seleccionó desarrollar el segmento de Turismo Romance, introduciendo las bodas de destino en un hotel de lujo en Canoa, Manabí Ecuador. Cabe recalcar que en la investigación realizada no existe la terminología, ni datos formales acerca de esta categoría de manera local, a diferencia de otros países, por ejemplo, México, en donde el turismo romance forma parte de uno de los sectores turísticos más explotados y por ende más lucrativos. 
Para poder encaminar de manera exitosa y justificada los esfuerzos económicos del sector, se plantean utilizar el análisis de conglomerados en el establecimiento de grupos objetivos que les parezca atractiva la opción de una boda de destino. Es de destacar, que este estudio se realizó posterior al terremoto que afectó a la región el 16 de abril de 2016, considerado en América Latina, como el tercer sismo más mortífero de los últimos 15 años, superado por el de Haití en enero de 2010, con entre 200.000 y 250.000 muertos, y los dos que sacudieron a El Salvador en enero y febrero de 2001, con 1.142 fallecidos. (El Telégrafo, 2016). Con base a lo expuesto, se establece como objetivo de este articulo proponer mediante criterios objetivos y psicográficos el grupo objetivo al cual dirigirse a través del análisis multivariable de tipo clúster.

\section{Desarrollo}

\section{Turismo Romance}

El turismo de romance, se refiere a: "turismo de aquellas parejas que efectúan un viaje para celebrar un motivo especial en su vida como: bodas, lunas de miel o renovación de votos". Asimismo, se consideran también en este segmento "los viajes de despedida de soltero, los baby moon (celebración de la concepción de un bebé) y family moons (cuando los novios tienen hijos y ellos forman parte del viaje por la boda o después de la boda)" (SECTUR, 2011). Es importante mencionar que los sub-segmentos del turismo de romance más lucrativos son: las bodas de destino y las lunas de miel.

\section{Boda de Destino}

Una boda es uno de los eventos más importantes en la vida de una pareja. No es solo para las personas que quieren compartir sus vidas juntas por primera vez, también puede ser una celebración de renovación de votos o aniversarios.

Básicamente, las bodas ya han sido consideradas como una clase de ritual que simboliza la transición de una pareja de un estado social a otro y de acuerdo a Edwards, esta clase de rito tiene diferentes características (Edwards, 1987); las cuales pueden apreciarse a través de imágenes típicas de ideales de un estatus social: verbalizaciones explícitas; como los votos de boda y discursos, así como actos simbólicos que se pueden encontrar en los procedimientos rituales. La razón por la que se comercializan es que han dejado de ser un festejo simple, centrándose más en los detalles de la recepción y no en el propósito fundamental de celebrar la boda. La comercialización o simplificación de las bodas depende de la planificación que haga la pareja del 
evento, ya que ellos saben lo que les gustaría representar en su matrimonio: una celebración de la unidad del amor o de la condición social.

Por lo general, el término boda simboliza el resultado del amor romántico, y es una muestra de un fuerte compromiso de votos de por vida. Por lo tanto, las bodas marcan el inicio del matrimonio (Leeds, 2002). De esta forma, representa un rito que formaliza la unión de dos personas que quieren hacer legal su relación (Appadurai, 1997), generando compromisos contractuales u obligaciones legales entre las partes contrayentes.

Leeds (2002) resumió que una boda pretende ser una unidad firme y natural, durante la celebración de un evento público y documentado. El término "bodas de destino", según el diccionario de la industria del viaje, es: "Una boda que tiene lugar en una ubicación distinta al de la casa de la novia y del novio, en un lugar popular de destino" (Travel Industry Dictionary, s.f.). Las bodas de destino tienen tres factores que las diferencian de las bodas tradicionales:

- Parámetros de planeación únicos: ya que las bodas de destino deben considerar una organización a larga distancia y tener que confiar en intermediarios y proveedores.

- $\quad$ El viaje como un importante componente de la boda. La pareja y algunos o todos los invitados tendrán que viajar, así que el transporte y hoteles se tendrán que considerar como parte de la planeación.

- Un evento de varios días. Contrario a una boda tradicional que es un evento de un día, las bodas de destino son eventos de mínimo dos días.

Los tres factores principales para las parejas para decidirse por una boda de destino son: presupuesto, atractivo del destino y el deseo de hacer algo único y especial. (Santillán, 2015). Según un estudio realizado en 2014 por The Knot, web especializada en bodas de destino, algunos de los factores que las parejas consideran un destino para casarse, en orden de importancia, son facilidad de acceso, seguridad, accesibilidad (el precio debe ser menor al del lugar de origen), el destino debe ser atractivo, variedad de actividades, variedad y calidad en hoteles, clima, hospitalidad, diversión, facilidad de organización y la gastronomía.

En cuanto a la preferencia sobre el tema de las bodas, el concepto de «temas», se refiere a la identificación recurrente de ideas, creencias y valores, dando un significado en cada uno de los grupos culturales. Dichos términos aplicados a las bodas, podrían hacer las cosas más emocionantes y memorables para la pareja, la familia y los invitados. Además, tener un tema, por tanto, puede ser una manera de honrar a las raíces de uno, y añade una inmensa carga emocional 
a la ceremonia (Chadiha, Leber, \& Veroff, 1998). Prácticamente, las bodas temáticas se refieren a la aplicación de un determinado modelo o concepto que guía a la ceremonia general.

Por otra parte, lo que hace el tema de la boda es la disposición de ciertos colores, símbolos históricos y todo lo que la pareja desee forme parte de la ceremonia, ya sean alimentos, objetos, vestuario, recuerdos y adornos adicionales sobre un tema en específico, integrar una mascota muy querida, hasta un género de música en especial, etcétera. La temática de la boda ya se puede considerar como un elemento esencial en la planeación y ejecución para que se dé un evento perfecto e inmemorable (Pollit, 2008).

Entonces, el producto de bodas de destino en Canoa, Ecuador consiste en un clúster integral de negocio que cubrirá totalmente las necesidades que se presentan en una boda.

Este producto se creará mediante la alianza estratégica de:

- $\quad$ Escenarios: Canoa

- Proveedores: Catering, menaje, mobiliarios, decoración, alimentos y bebidas, iluminación, sonido, etc. Es importante resaltar la gastronomía local, con platillos hechos a base de mariscos, hojas de plátano, salsas de cacahuates, dulces de Rocafuerte y los productos de decoración sostenibles como la paja toquilla, el mateancho (calabaza), las redes de pesca, los caracoles, la tagua (marfil vegetal) como elementos exóticos. También estarán a disposición del cliente opciones más "tradicionales".

- Transporte y hoteles: Pasajes aéreos, traslados y habitaciones con precios competitivos mediante la gestión de convenios corporativos diseñados para el segmento de bodas de destino.

- Tours: Debido a la cantidad de días que suelen estar los novios e invitados, se integrará la opción de tours tanto en Canoa como sus alrededores para brindar una experiencia completa.

\section{Análisis de Conglomerados}

El análisis clúster, conocido también como análisis de conglomerados, es una técnica multivariante de estadística que busca agrupar elementos logrando homogeneidad dentro de los grupos y diferencias con los otros. Tiene una importante aplicación en muchas áreas de investigación, sin embargo, junto con los beneficios del análisis clúster existen algunos inconvenientes, por ejemplo, calificado como descriptivo, ateórica y no inferencial. No tiene bases estadísticas sobre las cuales se puedan deducir inferencias para una población a partir de una muestra, es un método basado en criterios geométricos fundamentalmente, como una técnica 
exploratoria descriptiva pero no explicativa, ya que las soluciones no son únicas en la media en que la pertenencia al conglomerado para cualquier número de soluciones depende de muchos elementos elegidos mediante el procedimiento elegido. Por otra parte, la solución del clúster depende de la adición o destrucción de variables relevantes que pueden tener un impacto substancial en la solución resultante. (Fuente, 2011).

\section{Método de Ward}

El método de Ward une los casos buscando minimizar la varianza dentro de cada grupo. Para ello, se calcula en primer lugar, la medida de todas las variables en cada conglomerado. A continuación, se calcula la distancia entre cada caso y la media del conglomerado, sumando después las distancias entre todos los casos. Luego, se agrupan los conglomerados que generan menos aumentos en la suma de las distancias dentro de cada conglomerado. Este procedimiento crea grupos homogéneos y con tamaños similares.

\section{Metodología}

El estudio siguió una metodología de tipo descriptiva y bajo un enfoque cuantitativo, La población estuvo constituida por 162 encuestados que eran especialistas en organización de bodas, de los cuales 103 contestaron todas las preguntas, a partir de un muestro no probabilístico. Para la recolección de información se utilizó la técnica de la encuesta online con la plataforma LimeSurvey. La construcción del instrumento utilizado, se realizó a partir de las indagaciones con los organizadores de bodas, que permitió resumir la información que se necesitaba obtener para cumplir el objetivo planteado en el estudio. Se formularon las preguntas según la situación de la persona que esté contestándola, habiendo dos escenarios posibles:

- $\quad$ Personas que tienen planes de casarse

- $\quad$ Personas que se han casado hasta 5 años atrás.

A través del cuestionario, se recogieron respuestas que ayudaron a alcanzar el objetivo propuesto. La primera pregunta fue un filtro el cual determinaba qué tipo de cuestionario el encuestado iba a responder, ya que, según su situación, la formulación variaría. Para ello se utilizó el siguiente mimograma para explicar la lógica planteada para cada grupo de preguntas:

Para cada grupo de preguntas: 
Tabla 1.

Mimograma explicativo de la construcción del cuestionario.

\begin{tabular}{|c|c|c|c|c|c|c|}
\hline $\begin{array}{l}\text { GR } \\
\text { UPO }\end{array}$ & P. & \multicolumn{4}{|c|}{ FORMULACIÓN PREGUNTAS GENERALES } & TIPO DE RESPUESTA \\
\hline \multirow{5}{*}{$\begin{array}{l}\text { G00 } \\
1\end{array}$} & $\mathrm{P} 001$ & \multicolumn{4}{|c|}{ Con respecto a su boda, cuál de las siguientes alternativas describe mejor su situación } & LISTA RADIO \\
\hline & P002 & \multicolumn{4}{|c|}{ Según su opinión ¿cuál o cuáles serían la/s locación/es ideal/es para casarse? } & OPCIÓN MÚLTIPLE \\
\hline & \multicolumn{2}{|c|}{$\begin{array}{l}\text { FORMULACIÓN PREGUNTAS } \\
\text { CASADOS }\end{array}$} & \multirow{4}{*}{ G010 } & \multicolumn{2}{|r|}{$\begin{array}{l}\text { FORMULACIÓN PREGUNTAS } \\
\text { SOLTEROS }\end{array}$} & \\
\hline & $\mathrm{P} 003$ & $\begin{array}{l}\text { ¿Cuál fue la locación que eligió } \\
\text { para celebrar la boda? }\end{array}$ & & \multirow[b]{2}{*}{ P010 } & $\mathrm{N} / \mathrm{A}$ & LISTA RADIO \\
\hline & P004 & $\begin{array}{l}\text { ¿Cómo conoció acerca de la } \\
\text { locación que eligió para } \\
\text { celebrar la boda? }\end{array}$ & & & $\begin{array}{l}\text { ¿Cómo conoció acerca de la } \\
\text { locación en la que considera } \\
\text { celebrar la boda? }\end{array}$ & OPCIÓN MÚLTIPLE \\
\hline \multirow{2}{*}{$\begin{array}{l}\text { G00 } \\
4\end{array}$} & P005 & $\begin{array}{l}\text { ¿Quién estuvo a cargo de la } \\
\text { organización de la boda? }\end{array}$ & & P011 & $\begin{array}{l}\text { ¿Quién estará a cargo de la } \\
\text { organización de la boda? }\end{array}$ & $\begin{array}{l}\text { ENTRADA NUMÉRICA } \\
\text { MÚLTIPLE }\end{array}$ \\
\hline & P006 & $\begin{array}{l}\text { Indique la importancia de los } \\
\text { siguientes factores para elegir } \\
\text { este sitio }\end{array}$ & \multirow[t]{2}{*}{ G012 } & P012 & $\begin{array}{l}\text { Indique la importancia de los } \\
\text { siguientes factores para elegir ese } \\
\text { sitio }\end{array}$ & MATRIZ \\
\hline \multirow{2}{*}{$\begin{array}{l}\text { G00 } \\
6\end{array}$} & P007 & $\begin{array}{l}\text { ¿Cuántos invitados tuvieron en } \\
\text { su boda? }\end{array}$ & & P013 & $\begin{array}{l}\text { ¿Cuántos invitados tendrán en su } \\
\text { boda? }\end{array}$ & LISTA RADIO \\
\hline & P008 & $\begin{array}{l}\text { ¿Cómo Celebró su matrimonio } \\
\text { civil? }\end{array}$ & & P014 & ¿Celebrará su matrimonio civil? & LISTA RADIO \\
\hline $\begin{array}{l}\text { G00 } \\
8\end{array}$ & P009 & $\begin{array}{l}\text { ¿Cuál fue el total que usted } \\
\text { pagó globalmente en la } \\
\text { celebración de su boda (sin } \\
\text { contar vestido, peinado, } \\
\text { maquillaje, luna de miel)? }\end{array}$ & G014 & P015 & $\begin{array}{l}\text { ¿Qué presupuesto tiene pensado } \\
\text { usted utilizar globalmente en la } \\
\text { celebración de su boda (sin contar } \\
\text { vestido, peinado, maquillaje, luna de } \\
\text { miel) }\end{array}$ & LISTA RADIO \\
\hline \multirow{2}{*}{$\begin{array}{l}\text { G01 } \\
6\end{array}$} & P016 & \multicolumn{4}{|c|}{$\begin{array}{l}\text { Indique la importancia que tienen para Ud. los siguientes aspectos relativos a la } \\
\text { celebración de una boda }\end{array}$} & MATRIZ \\
\hline & P017 & $\begin{array}{l}\text { Califique del } 1 \text { al } 5 \mathrm{su} \\
\text { experiencia. }\end{array}$ & \multicolumn{3}{|l|}{ N/A } & MATRIZ \\
\hline \multirow{4}{*}{$\begin{array}{l}\mathrm{G} 01 \\
8\end{array}$} & P018 & \multicolumn{4}{|c|}{ ¿Cuál de los siguientes planes de boda es más de tu agrado? } & LISTA RADIO \\
\hline & $\mathrm{P} 19 \mathrm{~A}$ & Imagen $\mathrm{A}$ & & P20A & Imagen $\mathrm{A}$ & MATRIZ \\
\hline & P19B & Imagen B & G018 & P20B & Imagen B & MATRIZ \\
\hline & $\mathrm{P} 19 \mathrm{C}$ & Imagen $\mathrm{C}$ & & P20C & Imagen $\mathrm{C}$ & MATRIZ \\
\hline G02 & P021 & \multicolumn{4}{|c|}{ Reconoce alguna de las locaciones: Escriba su respuesta } & TEXTOS CORTOS \\
\hline
\end{tabular}


Análisis de conglomerados para la creación de un servicio: una aplicación en la hotelería

\begin{tabular}{|c|c|c|c|}
\hline 1 & & & \\
\hline \multirow{3}{*}{$\begin{array}{l}\text { G02 } \\
2\end{array}$} & P022 & ¿Conoce el hotel Canoa Beach en la playa de CANOA MANABÍ? & LISTA RADIO \\
\hline & P023 & ¿Ha visitado el hotel Canoa Beach? & OPCIÓN MÚLTIPLE \\
\hline & P024 & Podría indicar su opinión sobre su experiencia en el Hotel Canoa Beach & MATRIZ \\
\hline \multirow{4}{*}{$\begin{array}{l}\text { G02 } \\
5\end{array}$} & P025 & Edad & TEXTOS CORTOS \\
\hline & P026 & Nível de estúdios & LISTA RADIO \\
\hline & P027 & Sexo & LISTA RADIO \\
\hline & P028 & Residencia & LISTA RADIO \\
\hline
\end{tabular}

\section{Fuente: Elaboración propia.}

Así mismo, la información se descodifico a partir de la siguiente ficha técnica, presentada en la:

Imagen 1: Proceso codificación de la información del Cuestionario.

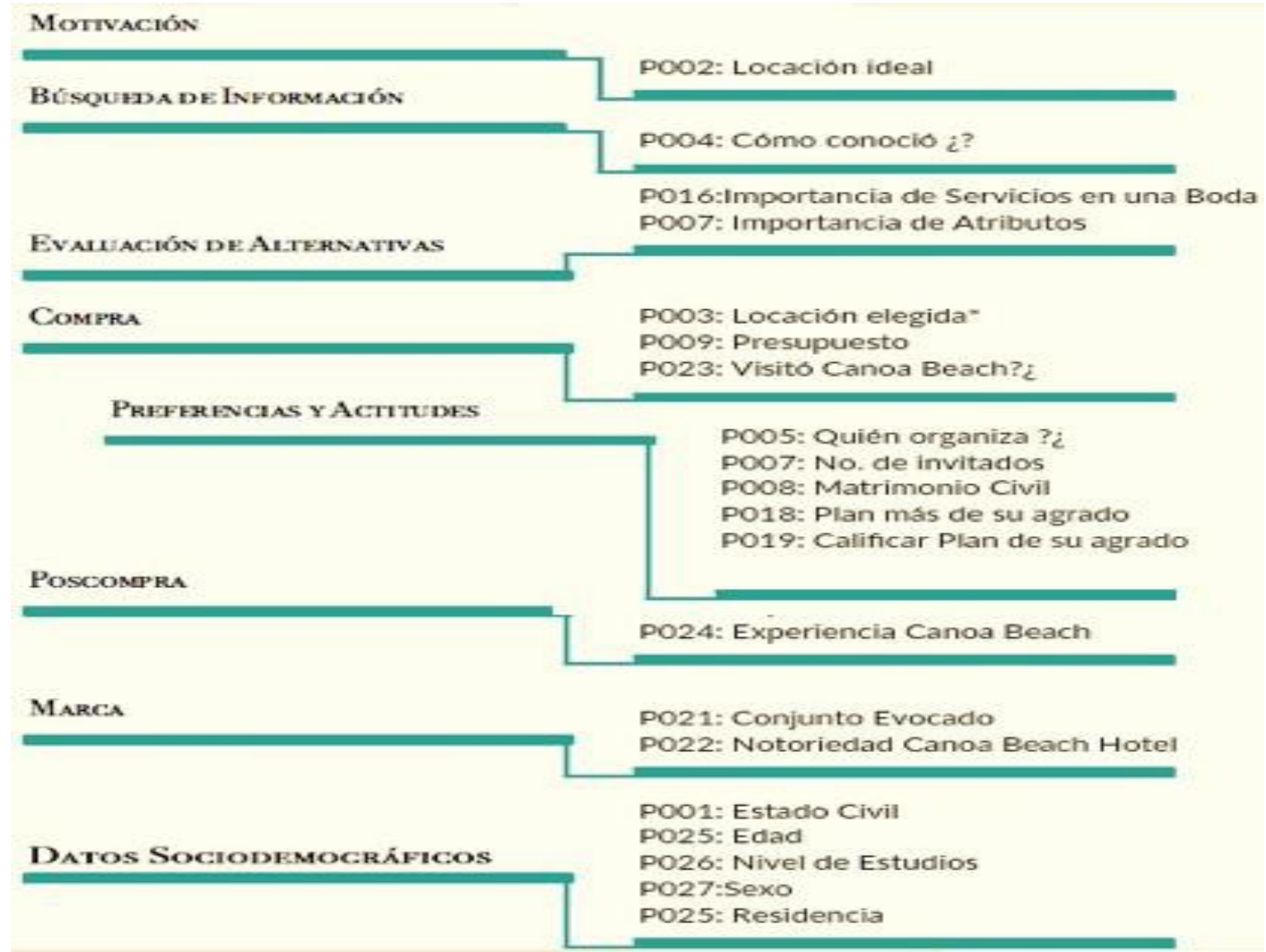

\section{Fuente: Elaboración propia}

Cabe señalar que la principal limitación de esta investigación de mercados fue que al contar con un perfil de entrevistado tan específico y al utilizar un muestreo de conveniencia, se limitó el tamaño de la muestra seleccionada ( $\mathrm{n}=103$ casos) haciendo de esta una muestra relativamente 
pequeña en comparación con el Universo. Además de que, al ser un estudio de carácter internacional, obligó a que el método de recolección sea vía internet, presentando limitaciones ya que impide conocer las reacciones reales de los encuestados y podrían generarse confusiones o malentendidos en los cuales no existe posibilidad de consultas acerca de dudas específicas.

\section{Resultados}

Mediante el análisis estadístico en SPSS, en este estudio se identificaron tres grupos por medio de un análisis clúster realizado sobre las contestaciones a la pregunta seis y la pregunta dieciséis. Dichas preguntas abarcan la importancia de los atributos determinantes para elegir una locación de bodas (P006) y los aspectos más importantes al realizar la boda (P016).

Lo primero que se identificó fueron 5 outliers, los cuales afectaban el resultado final, por lo cual se procedió a no tomarlos en cuenta para los conglomerados. El total de la muestra fueron 98 casos.

El análisis clúster se desarrolló siguiendo un método jerárquico el cual se analizó utilizando un proceso de agrupación del método de Ward, y como medida de similaridad se aplicó la distancia euclídea al cuadrado, obteniendo el historial de aglomeración. El resultado más óptimo fue el de tres clúster que se resume en la siguiente tabla:

\section{Tabla 2.}

\section{Clúster de grupos según importancia de atributos y aspectos}

\begin{tabular}{|l|l|l|l|l|}
\hline \multirow{2}{*}{ Variables } & \multicolumn{2}{|l|}{ Medias } & \multirow{2}{*}{ Sig. } \\
\cline { 2 - 5 } & $\begin{array}{l}\text { Prácticos } \\
(\mathrm{n}=37)\end{array}$ & $\begin{array}{l}\text { Emocionales } \\
(\mathrm{n}=35)\end{array}$ & $\begin{array}{l}\text { Exigentes } \\
(\mathrm{n}=26)\end{array}$ & \\
\hline Precio & 3,8 & 4,1 & 4,8 & 0,000 \\
\hline Comodidad & 3,9 & 4,2 & 4,8 & 0,000 \\
\hline Prestigio & 2,5 & 3,3 & 4,5 & 0,000 \\
\hline Ubicación & 3,8 & 4 & 4,6 & 0,005 \\
\hline Servicio & 4,2 & 4,5 & 4,8 & 0,005 \\
\hline Socio & 1,5 & 1,8 & 3,8 & 0,000 \\
\hline Emocional & 2,1 & 3,8 & 2,6 & 0,000 \\
\hline *Comida & 4,5 & 4,7 & 4,8 & 0,125 \\
\hline
\end{tabular}




\begin{tabular}{|l|l|l|l|l|} 
Bebidas & 2,1 & 3,8 & 2,6 & 0,000 \\
\hline Músicos & 3,8 & 4,4 & 4,8 & 0,000 \\
\hline *Decoración & 4,1 & 4,2 & 4,6 & 0,012 \\
\hline Meseros & 3,9 & 4,4 & 4,6 & 0,000 \\
\hline Fotografía & 4,2 & 4,5 & 4,8 & 0,004 \\
\hline *Clima & 4,2 & 4,3 & 4,5 & 0,337 \\
\hline Luna de miel & 3,7 & 4,6 & 4,5 & 0,001 \\
\hline Noche de bodas & 3,5 & 4,4 & 4,6 & 0,000 \\
\hline
\end{tabular}

*No presentan diferencias significativas

\section{Fuente: Elaboración propia}

Tal como se muestra en la tabla 2, aunque son grupos con prioridades distintas, todos coinciden en que el aspecto más importante de la boda es la comida.

Se denominaron "Prácticos", a los integrantes del primer grupo, caracterizados por darle una baja importancia a la comodidad $(3,9)$, precio/descuentos $(3,8)$, ubicación $(3,8)$ y prestigio, sin embargo exigen comida de alta calidad $(4,5)$ un buen servicio $(4,2)$, fotografías memorables $(4,2)$ y un clima agradable $(4,2)$. El factor emocional no incide en ellos $(2,1)$ tampoco el tener algún tipo de membresía $(1,5)$.

Por su parte los "Emocionales", además de exigir un buen banquete $(4,7)$ le brindan una alta importancia a la luna de miel $(4,6)$ así como a las fotografías que puedan tener como parte de sus recuerdos $(4,5)$. El prestigio $(3,3)$ y ser socio del lugar donde realizarían su evento $(1,8)$ es lo menos importante. De los tres grupos este tuvo una respuesta considerablemente más alta que los otros grupos acerca del aspecto emocional que puede tener una boda $(3,8)$.

En cuanto al tercer grupo, denominado como "Exigentes", tienen las mismas expectativas en cuanto a la importancia del precio/descuentos, comodidad, servicio, comida, músicos y fotografía $(4,8)$. En general sus expectativas son altas, a excepción de contar con una membresía $(3,8)$, a las bebidas $(2,6)$ e importancia del ámbito emocional $(2,6)$.

Los resultados del estudio de las características demográficas relacionados con el Perfil de los grupos, se presentan en la tabla 3. 
Tabla 3.

Características demográficas de los grupos identificados

\begin{tabular}{|c|c|c|c|c|c|c|c|c|c|c|}
\hline & \multirow{3}{*}{ Edad } & \multicolumn{3}{|c|}{ Prácticos } & \multicolumn{3}{|c|}{ Emocionales } & \multicolumn{3}{|c|}{ Exigentes } \\
\hline & & med & $\min$ & máx. & med & $\min$ & máx. & med & $\min$ & máx. \\
\hline & & 30 & 22 & 45 & 28 & 20 & 43 & 30 & 22 & 44 \\
\hline \multirow{5}{*}{ Situación: } & Casare prox. año & \multicolumn{3}{|c|}{$38,70 \%$} & \multicolumn{3}{|c|}{$35,50 \%$} & \multicolumn{3}{|c|}{$25,80 \%$} \\
\hline & Casaré 6 meses & \multicolumn{3}{|c|}{$50,00 \%$} & \multicolumn{3}{|c|}{$25,00 \%$} & \multicolumn{3}{|c|}{$25,00 \%$} \\
\hline & Menos 1 año cas. & \multicolumn{3}{|c|}{$25,00 \%$} & \multicolumn{3}{|c|}{$25,00 \%$} & \multicolumn{3}{|c|}{$50,00 \%$} \\
\hline & Entre 1 y 3 casado & \multicolumn{3}{|c|}{$16,70 \%$} & \multicolumn{3}{|c|}{$50,00 \%$} & \multicolumn{3}{|c|}{$33,30 \%$} \\
\hline & Entre 3 y 5 casado & \multicolumn{3}{|c|}{$38,90 \%$} & \multicolumn{3}{|c|}{$38,90 \%$} & \multicolumn{3}{|c|}{$22,20 \%$} \\
\hline \multirow{6}{*}{ Otro Residencia } & Cuenca & \multicolumn{3}{|c|}{$2,70 \%$} & \multicolumn{3}{|c|}{$8,60 \%$} & \multicolumn{3}{|c|}{$0,00 \%$} \\
\hline & Guayaquil & \multicolumn{3}{|c|}{$5,40 \%$} & \multicolumn{3}{|c|}{$5,70 \%$} & \multicolumn{3}{|c|}{$15,40 \%$} \\
\hline & Manta & \multicolumn{3}{|c|}{$24,30 \%$} & \multicolumn{3}{|c|}{$34,30 \%$} & \multicolumn{3}{|c|}{$38,50 \%$} \\
\hline & Otro & \multicolumn{3}{|c|}{$10,80 \%$} & \multicolumn{3}{|c|}{$14,30 \%$} & 19,2 & & \\
\hline & Portoviejo & $5,40^{\circ}$ & & & $8,60^{\circ}$ & & & 3,80 & & \\
\hline & Quito & 51,40 & & & 28,60 & & & 23,1 & & \\
\hline & FEMENINO & 41,90 & & & 63,60 & & & 24,2 & & \\
\hline & MASCULINO & 34,50 & & & 36,40 & & & 24,1 & & \\
\hline & Primaria & $0,00^{\circ}$ & & & $0,00^{\circ}$ & & & 0,00 & & \\
\hline & Bachillerato & $2,70^{\circ}$ & & & $5,70^{\circ}$ & & & 15,4 & & \\
\hline & Tecnólogo & $2,70^{\circ}$ & & & $2,90^{\circ}$ & & & 3,80 & & \\
\hline & Lic./ING & 51,40 & & & 48,60 & & & 61,5 & & \\
\hline & Máster & 37,80 & & & 40,00 & & & 15,4 & & \\
\hline & Doctorado & $5,40^{\circ}$ & & & $2,90^{\circ}$ & & & 3,80 & & \\
\hline
\end{tabular}

\section{Fuente: Elaboración propia}

El grupo de los prácticos son mujeres y hombres con una edad media de 30 años, mínima de 22 y máxima de 45 . La mitad de ellos se casarán en seis meses, representan el 51\% de la muestra que vive en Quito. El 51,40\% del total del grupo cuenta con título de licenciatura, el 37,80\% de 
máster y 5,80\% con doctorado. Los emocionales son mujeres y hombres con una edad media de 28 años, siendo la menor 20 y la mayor 43. Aunque en este grupo existe mayor concentración de personas que ya se han casado $(37,97 \%$ de la muestra total), existe un segmento importante del total de la muestra que se casarán el próximo año $(35,50 \%)$ y otro que se casarán en 6 meses (25\%). En su mayoría residen en Manta (34,30\%) y en Quito $(28,60 \%)$. Cuentan con una educación de tercer nivel $(48,60 \%)$ y máster (40\%). En cuanto a los exigentes son mujeres y hombres con una edad media de 30 años, mínima de 22 y máxima de 44. Ellos comprenden al $50 \%$ de la muestra que está casada menos de un año, ellos son de Manta (38,50\%), Quito(23,10\%) y otras localidades de Ecuador (19,20\%) y sus estudios comprenden un 61,50\% en grado o ingeniería, un $15,40 \%$ tienen titulación de máster y al igual que un $15,40 \%$ son de bachillerato.

\section{Tabla 4.}

Aspectos más relevantes en cuanto al estudio de cada uno de los grupos:

\begin{tabular}{|c|c|c|c|}
\hline & PRÁCTICOS & EMOCIONALES & EXIGENTES \\
\hline ¿Quiénes? & $\begin{array}{l}\text { Parejas estables, por } \\
\text { casarse entre } 6 \text { meses o } \\
\text { un año. }\end{array}$ & $\begin{array}{l}\text { Llevan casados entre } 1 \text { y } 5 \\
\text { años, se casarán el otro } \\
\text { año }\end{array}$ & $\begin{array}{l}\text { Llevan casados menos } \\
\text { de un año }\end{array}$ \\
\hline ¿Dónde? & Quito, Manta & Manta, Quito & Manta, Guayaquil, Quito \\
\hline ¿Estudios? & $\begin{array}{l}\text { Licenciatura o } \\
\text { ingeniería/Máster }\end{array}$ & $\begin{array}{l}\text { Licenciatura o } \\
\text { ingeniería/Máster }\end{array}$ & $\begin{array}{l}\text { Licenciatura o } \\
\text { ingeniería/Máster }\end{array}$ \\
\hline Búsqueda & $\begin{array}{l}\text { Ir a una boda, sugerencia } \\
\text { de amigos }\end{array}$ & Internet & Internet \\
\hline Lo más importante & $\begin{array}{l}\text { Comida, servicio, } \\
\text { fotografía y clima }\end{array}$ & $\begin{array}{l}\text { Comida, luna de miel y } \\
\text { fotografías }\end{array}$ & $\begin{array}{l}\text { Precio, comodidad, } \\
\text { servicio, comida, } \\
\text { decoración, fotografía }\end{array}$ \\
\hline Lo menos importante & $\begin{array}{l}\text { Socio, emocional, } \\
\text { bebidas }\end{array}$ & $\begin{array}{l}\text { Ser socio, que el lugar } \\
\text { tenga prestigio }\end{array}$ & $\begin{array}{l}\text { Emocional, Bebidas, ser } \\
\text { socio }\end{array}$ \\
\hline Presupuesto & $\begin{array}{l}\text { Menos de \$3000/ \$3.001- } \\
5.000\end{array}$ & $\$ 3.001-5.000$ & $\$ 3.001-8.000$ \\
\hline Invitados & $91-180$ & Hasta 90 & $91-180$ \\
\hline ¿Civil? & Cena íntima & Cena íntima & $\begin{array}{l}\text { Cena íntima, entre } 13 \text { y } 25 \\
\text { personas }\end{array}$ \\
\hline ¿Quién Organiza? & Encuestado, familiares, & Encuestado, familiares, & Encuestado, Wedding \\
\hline
\end{tabular}




\begin{tabular}{l|l|l|l|}
\multirow{2}{*}{ ¿Boda ideal? } & incluida & wedding planner & planner, familiares \\
\cline { 2 - 4 } \multirow{2}{*}{ ¿En qué lugar } & Playa $62,16 \%$ & Playa $66,71 \%$ & Playa $88,48 \%$ \\
\cline { 2 - 4 } & Decamerón & Canoa Beach Hotel & Hotel Oro Verde \\
\cline { 2 - 4 } & $\begin{array}{l}\text { Alta, varios aspectos con } \\
\text { pequeñas diferencias }\end{array}$ & $\begin{array}{l}\text { Alta, pocos aspectos con } \\
\text { pequeñas diferencias }\end{array}$ & $\begin{array}{l}\text { Muy alta, casi todos los } \\
\text { aspectos con pequeñas } \\
\text { diferencias. }\end{array}$ \\
\hline
\end{tabular}

\section{Fuente: Elaboración propia}

La tabla 4, presenta de forma esquemática los aspectos más relevantes en cuanto al estudio de cada uno de los grupos investigados.

\section{Conclusión}

Las parejas prácticas se fijan en general en la comida, servicio, fotografía y clima de su boda, sin importarles los aspectos emocionales. Quienes están en el grupo de parejas emocionales, además de la comida y fotografía que se repite, se preocupan por la luna de miel y tienen en cuenta la carga emocional, ellos no invitan a tantas personas y les atrae el romanticismo. En el caso de las parejas exigentes, esperan una alta calidad en todos los aspectos, y es probable que, aunque no se alcance sus expectativas, tendrán una alta satisfacción y muestran una alta implicación. Aunque gustan de las bodas de la playa, eligen locaciones más convencionales para evitar el riesgo de que algo salga mal, aunque en el fondo quisieran una boda descomplicada y romántica, por lo que existe una dirección del esfuerzo hacia el grupo de los emocionales y los prácticos que son los grupos que muestran mayor aceptación al, servicio prestado.

\section{Referencias Bibliográficas}

Appadurai, A. (1997). Consumption, duration, and history. In D. Palumbo-Liu y H.U. Gumbrecht. Standford, California: Stanford University Press.

Chadiha, L., Leber, D., \& Veroff, J. (1998). Newlywed's narrative themes: meaning in the first year of marriage for African American and white couples. Journal of Comparative Family Studies.

Diario el Universo. (s/f). Matrimonio: Noticias de actualidad sobre Matrimonio . Recuperado de : https://www.eluniverso.com/tema/matrimonio

Edwards, W. (1987). The Commercialized Wedding as Ritual: A Window on Social Values. Journal of Japanese Studies., 13. 
El Telégrafo. (30 de mayo de 2016). La ayuda y el turismo primaron en canoa. (R. Manabí, Ed.) La ayuda y el turismo primaron en Manabí, pág. 12. Obtenido de http://www.eltelegrafo.com.ec/noticias/ecuador/3/la-ayuda-y-el-turismo-primaron-encanoa

Fuente, S. d. (2011). Análisis Conglomerados. Madrid: UAM.

Leeds, H. (2002). Weeding as Text: Communicating Cultural Identities through Ritual. Mahwah. Mahwah, NJ: Lawrence Erlbaum Associates.

Pollit, D. (2008). Developing a Theme for your wedding. Recuperado el 02 de 14 de 2017, de America's Top Wedding Links: http://www.topweddinglinks.com/Articles/weddingthemed.html

Santillán, F. (2015). Procesos e innovaciones en la educación a nivel superior en Latinoamérica. España: Centro de Estudios e Investigaciones para el Desarrollo Docente (CENID). SECTUR. (2011). Subsecretaria de Planeación Turística. Recuperado el 14 de 2 de 2017, de RESUMEN EJECUTIVO: http://ictur.sectur.gob.mx/descargas/Publicaciones/Boletin/cedoc2012/ cedoc2011/EstudioRomance.pdf

Travel Industry Dictionary. (s.f.). Travel Industry Dictionary . Recuperado el 14 de 02 de 2017, de http://www. travel-industry-dictionary.com/destination-wedding.html

\section{Anexos}

Técnicas de descripción general de las variables:

Frecuencias: Análisis univariante que permite analizar de forma descriptiva las variables nominales mediante la generación de tablas con sus porcentajes.

Medias: análisis univariante realizado con variables continuas o numéricas que permite obtener información del valor central de las respuestas.

Técnicas de comprobación de independencia:

Análisis de la varianza (Anova): Técnica de análisis bivariante usada para contrastar los test de hipótesis comparando medias para saber si existe relación entre ellos.

Técnicas de generación de grupo:

Análisis Clúster: Técnica multivariante que se centra en formar grupos homogéneos entre sí y heterogéneos entre los distintos grupos, consiguiéndose grupos a través de las variables continúas 
analizadas. A continuación, detallaremos los datos obtenidos durante la descripción de los clúster:

ean difference is significant at the 0.05 level.

Case Processing Summarya

\begin{tabular}{|c|c|c|c|c|c|}
\hline \multicolumn{2}{|c|}{ Cases } \\
\hline \multicolumn{2}{|c|}{ Valid } & \multicolumn{2}{c|}{ Missing } & \multicolumn{2}{c|}{ Total } \\
\hline N & Percent & N & Percent & N & Percent \\
\hline 98 & 100,0 & 0 & 0,0 & 98 & 100,0 \\
\hline
\end{tabular}

\section{Ward Method}

\begin{tabular}{|c|c|c|c|c|c|}
\hline \multicolumn{2}{|c|}{} & Frequency & Percent & Valid Percent & Cumulative Percent \\
\hline \multirow{3}{*}{ Valid } & Prácticos & 37 & 37,8 & 37,8 & 37,8 \\
\cline { 2 - 6 } & Emocionales & 35 & 35,7 & 35,7 & 73,5 \\
\cline { 2 - 6 } & Exigentes & 26 & 26,5 & 26,5 & 100,0 \\
\cline { 2 - 6 } & Total & 98 & 100,0 & 100,0 & \\
\hline
\end{tabular}

\section{Descriptives}

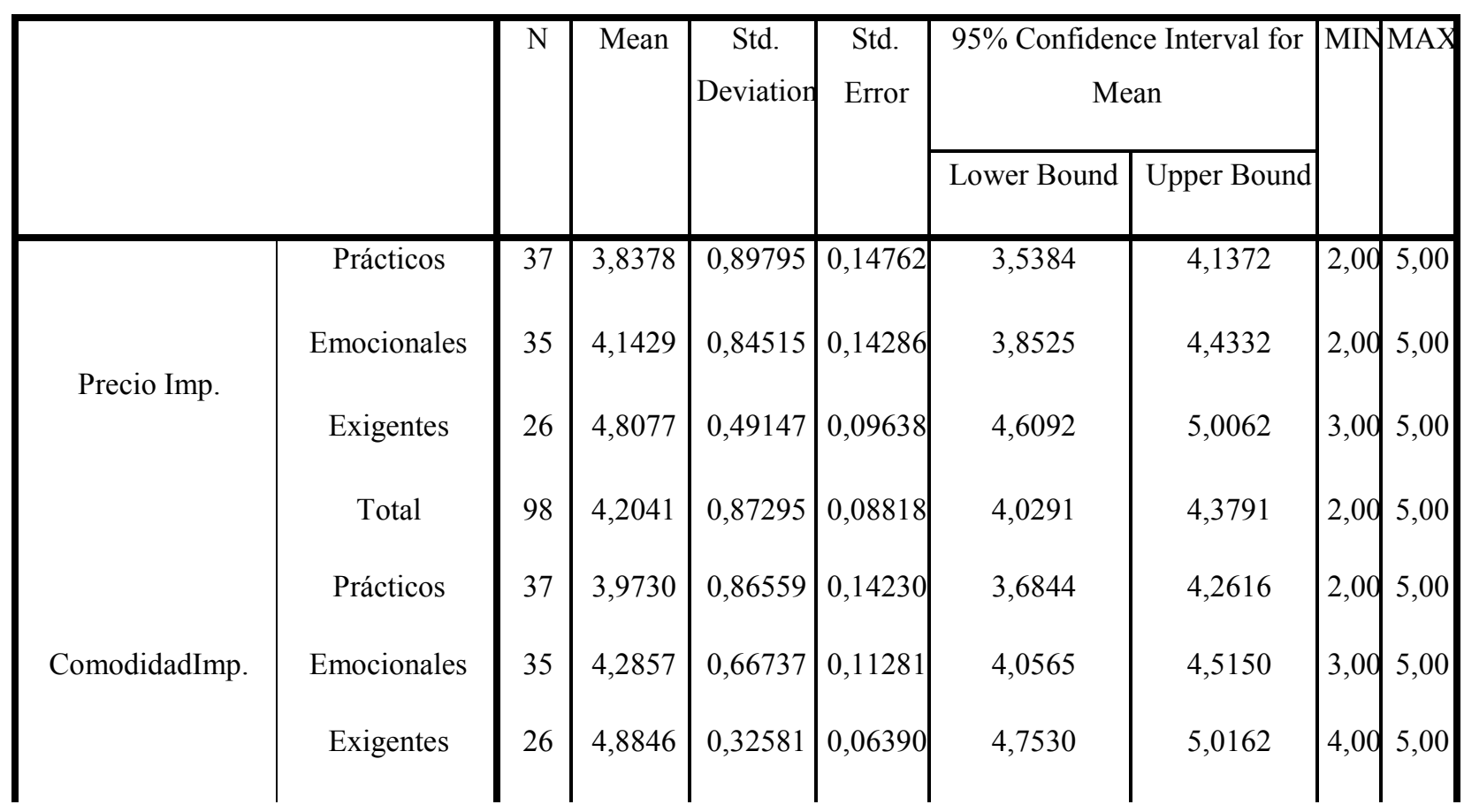




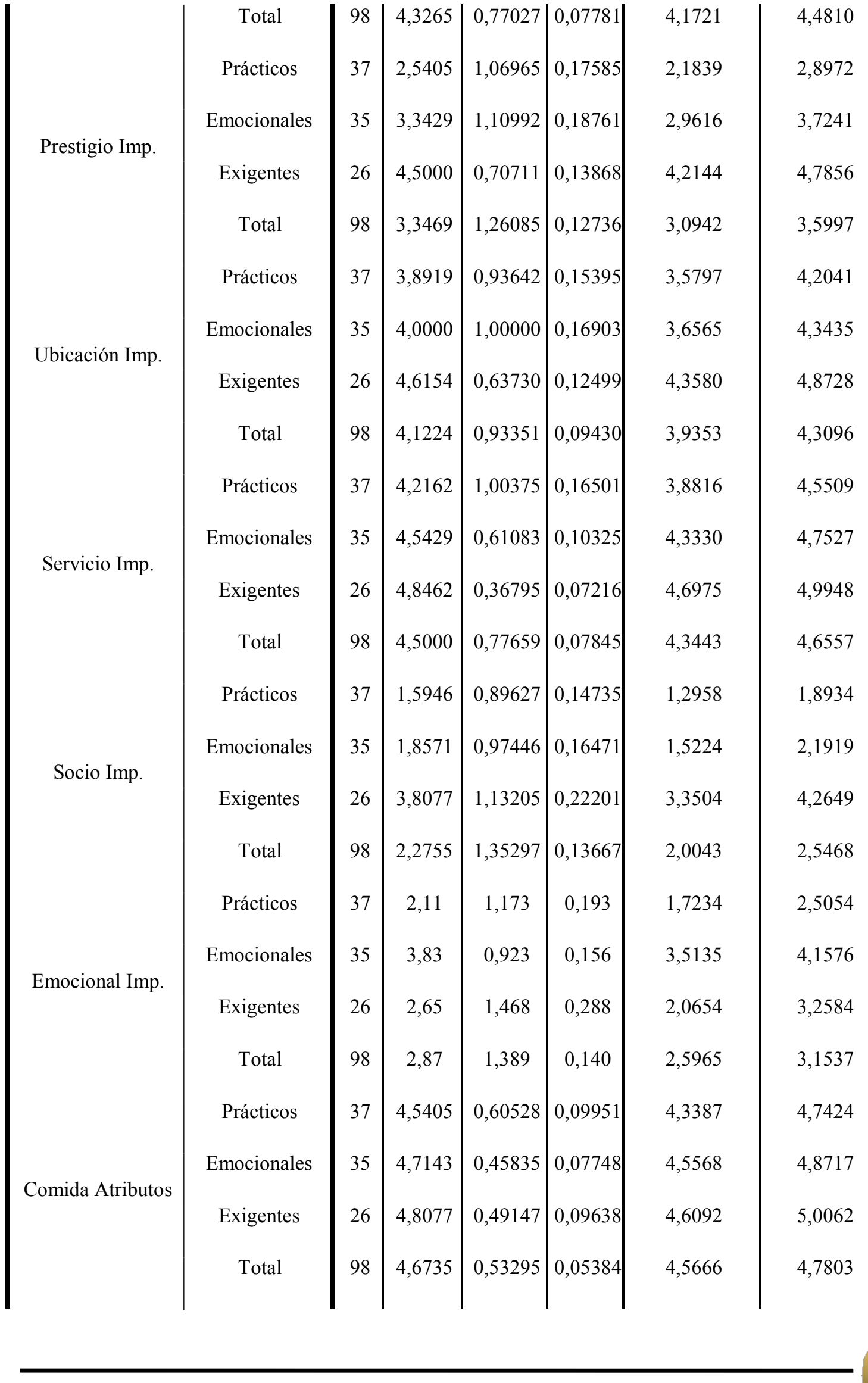




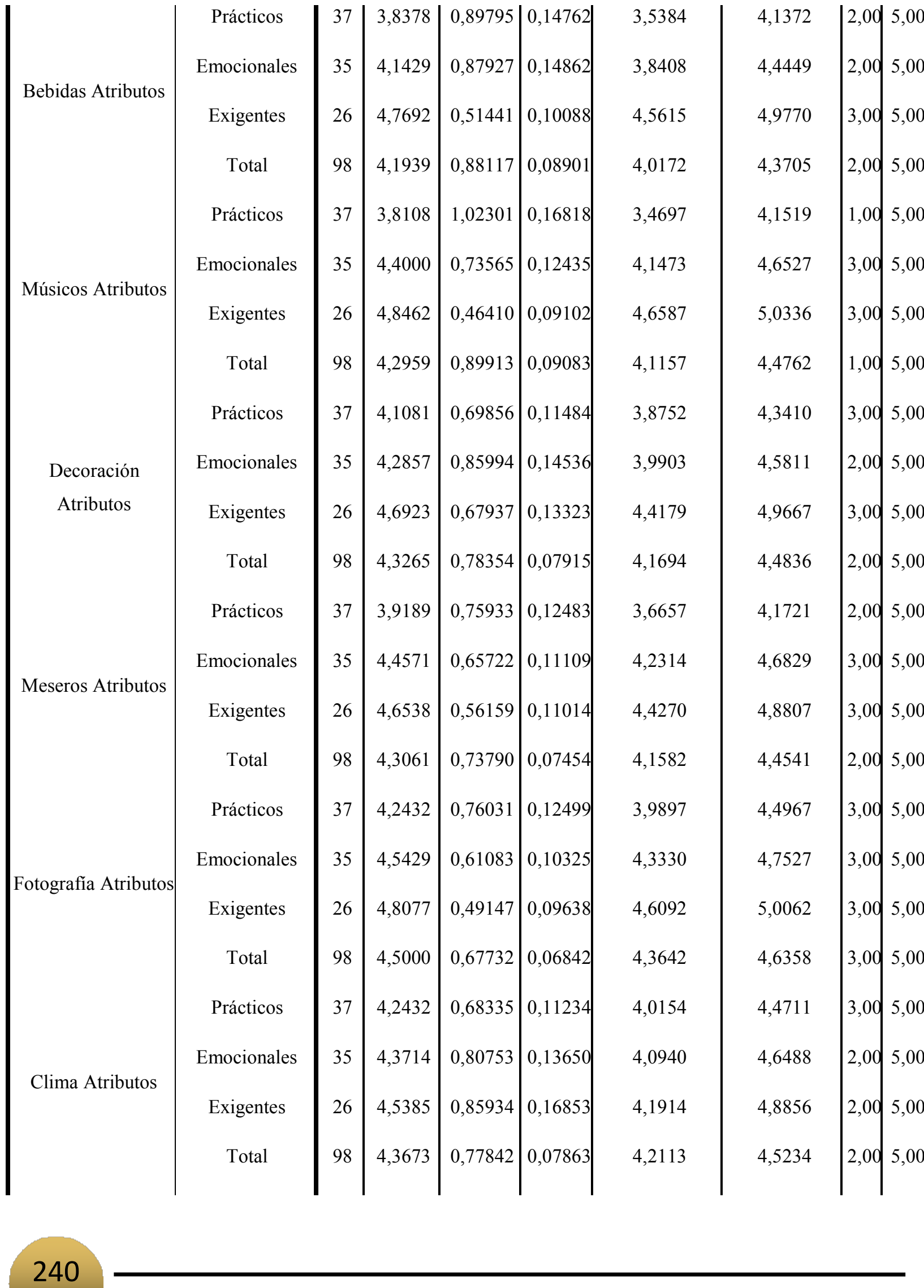

Pol. Con. (Edición núm. 29) Vol. 4, No 1, enero 2019, pp. 223-251, ISSN: 2550 - 682X 
Análisis de conglomerados para la creación de un servicio: una aplicación en la hotelería

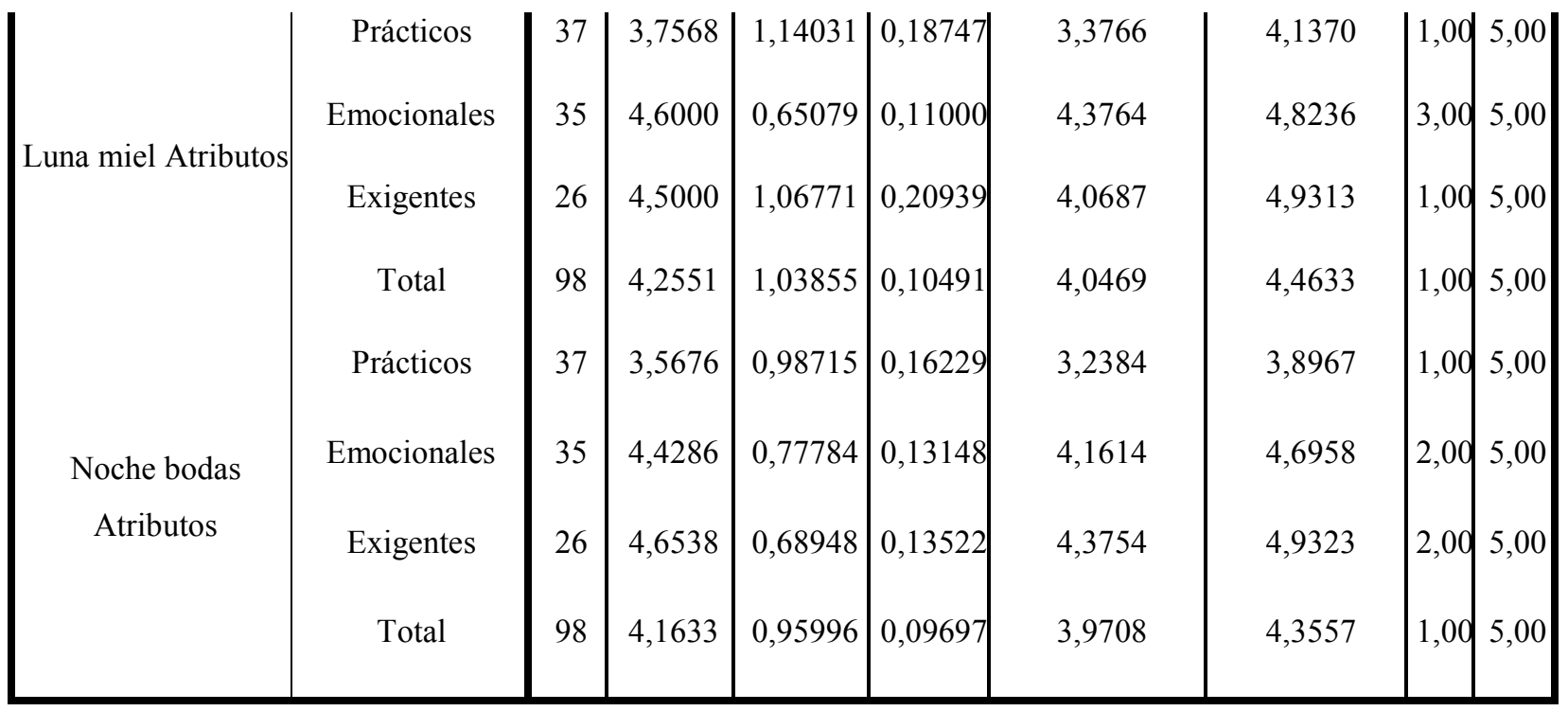

Anova

\begin{tabular}{|c|c|c|c|c|c|c|}
\hline & & Sum of Squares & $\mathrm{df}$ & Mean Square & $\mathrm{F}$ & Sig. \\
\hline \multirow{3}{*}{ Precio Imp. } & Between Groups & 14,567 & 2 & 7,284 & 11,658 & 0,000 \\
\hline & Within Groups & 59,351 & 95 & 0,625 & & \\
\hline & Total & 73,918 & 97 & & & \\
\hline \multirow{3}{*}{ ComodidadImp. } & Between Groups & 12,781 & 2 & 6,391 & 13,561 & 0,000 \\
\hline & Within Groups & 44,770 & 95 & 0,471 & & \\
\hline & Total & 57,551 & 97 & & & \\
\hline \multirow{3}{*}{ Prestigio Imp. } & Between Groups & 58,629 & 2 & 29,315 & 29,138 & 0,000 \\
\hline & Within Groups & 95,575 & 95 & 1,006 & & \\
\hline & Total & 154,204 & 97 & & & \\
\hline \multirow{3}{*}{ Ubicación Imp. } & Between Groups & 8,809 & 2 & 4,405 & 5,526 & 0,005 \\
\hline & Within Groups & 75,721 & 95 & 0,797 & & \\
\hline & Total & 84,531 & 97 & & & \\
\hline \multirow{2}{*}{ Servicio Imp. } & Between Groups & 6,159 & 2 & 3,080 & 5,590 & 0,005 \\
\hline & Within Groups & 52,341 & 95 & 0,551 & & \\
\hline
\end{tabular}




\begin{tabular}{|c|c|c|c|c|c|c|}
\hline & Total & 58,500 & 97 & & & \\
\hline \multirow{3}{*}{ Socio Imp. } & Between Groups & 84,318 & 2 & 42,159 & 42,953 & 0,000 \\
\hline & Within Groups & 93,243 & 95 & 0,982 & & \\
\hline & Total & 177,561 & 97 & & & \\
\hline \multirow{3}{*}{ Emocional Imp. } & Between Groups & 54,852 & 2 & 27,426 & 19,675 & 0,000 \\
\hline & Within Groups & 132,424 & 95 & 1,394 & & \\
\hline & Total & 187,276 & 97 & & & \\
\hline \multirow{3}{*}{ Comida Atributos } & Between Groups & 1,181 & 2 & 0,590 & 2,126 & 0,125 \\
\hline & Within Groups & 26,371 & 95 & 0,278 & & \\
\hline & Total & 27,551 & 97 & & & \\
\hline \multirow{3}{*}{ Bebidas Atributos } & Between Groups & 13,388 & 2 & 6,694 & 10,269 & 0,000 \\
\hline & Within Groups & 61,928 & 95 & 0,652 & & \\
\hline & Total & 75,316 & 97 & & & \\
\hline \multirow{3}{*}{ Músicos Atributos } & Between Groups & 16,958 & 2 & 8,479 & 13,106 & 0,000 \\
\hline & Within Groups & 61,460 & 95 & 0,647 & & \\
\hline & Total & 78,418 & 97 & & & \\
\hline \multirow{3}{*}{ Decoración Atributos } & Between Groups & 5,302 & 2 & 2,651 & 4,643 & 0,012 \\
\hline & Within Groups & 54,249 & 95 & 0,571 & & \\
\hline & Total & 59,551 & 97 & & & \\
\hline \multirow{3}{*}{ Meseros Atributos } & Between Groups & 9,489 & 2 & 4,745 & 10,403 & 0,000 \\
\hline & Within Groups & 43,327 & 95 & 0,456 & & \\
\hline & Total & 52,816 & 97 & & & \\
\hline \multirow{3}{*}{ Fotografia Atributos } & Between Groups & 4,965 & 2 & 2,483 & 5,965 & 0,004 \\
\hline & Within Groups & 39,535 & 95 & 0,416 & & \\
\hline & Total & 44,500 & 97 & & & \\
\hline
\end{tabular}


Análisis de conglomerados para la creación de un servicio: una aplicación en la hotelería

\begin{tabular}{|c|c|c|c|c|c|c|}
\hline \multirow{4}{*}{ Clima Atributos } & Between Groups & 1,332 & 2 & 0,666 & 1,101 & 0,337 \\
\cline { 2 - 7 } & Within Groups & 57,444 & 95 & 0,605 & & \\
\cline { 2 - 7 } & Total & 58,776 & 97 & & & \\
\hline \multirow{3}{*}{ Luna miel Atributos } & Between Groups & 14,912 & 2 & 7,456 & 7,895 & 0,001 \\
\cline { 2 - 7 } & Within Groups & 89,711 & 95 & 0,944 & & \\
\cline { 2 - 7 } & Total & 104,622 & 97 & & & \\
\hline \multirow{2}{*}{$\begin{array}{c}\text { Noche bodas } \\
\text { Atributos }\end{array}$} & Between Groups & 21,851 & 2 & 10,925 & 15,368 & 0,000 \\
\cline { 2 - 7 } & Within Groups & 67,537 & 95 & 0,711 & & \\
\cline { 2 - 7 } & Total & 89,388 & 97 & & & \\
\hline
\end{tabular}

Multiple Comparisons

\begin{tabular}{|c|c|c|c|c|c|c|c|c|}
\hline \multirow{2}{*}{\multicolumn{2}{|c|}{ Dependent Variable }} & \multirow[t]{2}{*}{$\begin{array}{l}\text { (I) Ward } \\
\text { Method }\end{array}$} & \multirow[t]{2}{*}{$\begin{array}{l}\text { (J) Ward } \\
\text { Method }\end{array}$} & \multirow{2}{*}{$\begin{array}{c}\text { Mean } \\
\text { Difference } \\
\text { (I-J) }\end{array}$} & \multirow[t]{2}{*}{ Std. Error } & \multirow[t]{2}{*}{ Sig. } & \multicolumn{2}{|c|}{$\begin{array}{c}\text { 95\% Confidence } \\
\text { Interval }\end{array}$} \\
\hline & & & & & & & $\begin{array}{l}\text { Lower } \\
\text { Bound }\end{array}$ & Upper Bound \\
\hline \multirow{13}{*}{ Precio Imp. } & \multirow{6}{*}{ Gabriel } & \multirow{2}{*}{ Prácticos } & Emocionales & $-0,30502$ & 0,18637 & 0,281 & $-0,7577$ & 0,1476 \\
\hline & & & Exigentes & $-0,96985^{*}$ & 0,20227 & 0,000 & $-1,4593$ & $-0,4804$ \\
\hline & & \multirow{2}{*}{ Emocionales } & Prácticos & 0,30502 & 0,18637 & 0,281 & $-0,1476$ & 0,7577 \\
\hline & & & Exigentes & $-0,66484^{*}$ & 0,20464 & 0,005 & $-1,1606$ & $-0,1691$ \\
\hline & & \multirow{2}{*}{ Exigentes } & Prácticos & $0,96985^{*}$ & 0,20227 & 0,000 & 0,4804 & 1,4593 \\
\hline & & & Emocionales & $0,66484^{*}$ & 0,20464 & 0,005 & 0,1691 & 1,1606 \\
\hline & \multirow{7}{*}{$\begin{array}{l}\text { Games- } \\
\text { Howell }\end{array}$} & \multirow{3}{*}{ Prácticos } & Emocionales & $-0,30502$ & 0,20543 & 0,304 & $-0,7969$ & 0,1869 \\
\hline & & & & & & & & \\
\hline & & & Exigentes & $-0,96985^{*}$ & 0,17630 & 0,000 & $-1,3939$ & $-0,5458$ \\
\hline & & & Prácticos & 0,30502 & 0,20543 & 0,304 & $-0,1869$ & 0,7969 \\
\hline & & & Exigentes & $-0,66484 *$ & 0,17233 & 0,001 & $-1,0797$ & $-0,2500$ \\
\hline & & & Prácticos & $0,96985^{*}$ & 0,17630 & 0,000 & 0,5458 & 1,3939 \\
\hline & & & Emocionales & $0,66484^{*}$ & 0,17233 & 0,001 & 0,2500 & 1,0797 \\
\hline & & & & & & & & \\
\hline
\end{tabular}


María Claudia Rivera Prado, Arturo Damián Rodríguez Zambrano, Sandy Lisbeth Hormaza Villafuerte

\begin{tabular}{|c|c|c|c|c|c|c|c|c|}
\hline \multirow{12}{*}{ ComodidadImp. } & \multirow{6}{*}{ Gabriel } & \multirow{2}{*}{ Prácticos } & Emocionales & $-0,31274$ & 0,16187 & 0,159 & $-0,7059$ & 0,0804 \\
\hline & & & Exigentes & $-0,91164 *$ & 0,17568 & 0,000 & $-1,3367$ & $-0,4866$ \\
\hline & & \multirow{2}{*}{ Emocionales } & Prácticos & 0,31274 & 0,16187 & 0,159 & $-0,0804$ & 0,7059 \\
\hline & & & Exigentes & $-0,59890^{*}$ & 0,17774 & 0,003 & $-1,0294$ & $-0,1684$ \\
\hline & & \multirow{2}{*}{ Exigentes } & Prácticos & $0,91164^{*}$ & 0,17568 & 0,000 & 0,4866 & 1,3367 \\
\hline & & & Emocionales & $0,59890 *$ & 0,17774 & 0,003 & 0,1684 & 1,0294 \\
\hline & \multirow{6}{*}{$\begin{array}{l}\text { Games- } \\
\text { Howell }\end{array}$} & \multirow{2}{*}{ Prácticos } & Emocionales & $-0,31274$ & 0,18159 & 0,204 & $-0,7479$ & 0,1225 \\
\hline & & & Exigentes & $-0,91164 *$ & 0,15599 & 0,000 & $-1,2886$ & $-0,5347$ \\
\hline & & \multirow{2}{*}{ Emocionales } & Prácticos & 0,31274 & 0,18159 & 0,204 & $-0,1225$ & 0,7479 \\
\hline & & & Exigentes & $-0,59890^{*}$ & 0,12965 & 0,000 & $-0,9117$ & $-0,2861$ \\
\hline & & \multirow{2}{*}{ Exigentes } & Prácticos & $0,91164^{*}$ & 0,15599 & 0,000 & 0,5347 & 1,2886 \\
\hline & & & Emocionales & $0,59890^{*}$ & 0,12965 & 0,000 & 0,2861 & 0,9117 \\
\hline \multirow{12}{*}{ Prestigio Imp. } & \multirow{6}{*}{ Gabriel } & \multirow{2}{*}{ Prácticos } & Emocionales & $-0,80232 *$ & 0,23651 & 0,003 & $-1,3767$ & $-0,2279$ \\
\hline & & & Exigentes & $-1,95946^{*}$ & 0,25668 & 0,000 & $-2,5805$ & $-1,3384$ \\
\hline & & \multirow{2}{*}{ Emocionales } & Prácticos & $0,80232 *$ & 0,23651 & 0,003 & 0,2279 & 1,3767 \\
\hline & & & Exigentes & $-1,15714^{*}$ & 0,25969 & 0,000 & $-1,7862$ & $-0,5281$ \\
\hline & & \multirow{2}{*}{ Exigentes } & Prácticos & $1,95946^{*}$ & 0,25668 & 0,000 & 1,3384 & 2,5805 \\
\hline & & & Emocionales & $1,15714^{*}$ & 0,25969 & 0,000 & 0,5281 & 1,7862 \\
\hline & \multirow{6}{*}{$\begin{array}{l}\text { Games- } \\
\text { Howell }\end{array}$} & \multirow{2}{*}{ Prácticos } & Emocionales & $-0,80232 *$ & 0,25714 & 0,007 & $-1,4182$ & $-0,1865$ \\
\hline & & & Exigentes & $-1,95946^{*}$ & 0,22395 & 0,000 & $-2,4975$ & $-1,4214$ \\
\hline & & \multirow{2}{*}{ Emocionales } & Prácticos & $0,80232^{*}$ & 0,25714 & 0,007 & 0,1865 & 1,4182 \\
\hline & & & Exigentes & $-1,15714^{*}$ & 0,23330 & 0,000 & $-1,7183$ & $-0,5959$ \\
\hline & & \multirow{2}{*}{ Exigentes } & Prácticos & $1,95946^{*}$ & 0,22395 & 0,000 & 1,4214 & 2,4975 \\
\hline & & & Emocionales & $1,15714^{*}$ & 0,23330 & 0,000 & 0,5959 & 1,7183 \\
\hline Ubicación Imp. & Gabriel & Prácticos & Emocionales & $-0,10811$ & 0,21051 & 0,939 & $-0,6194$ & 0,4032 \\
\hline
\end{tabular}

\section{4}

Pol. Con. (Edición núm. 29) Vol. 4, No 1, enero 2019, pp. 223-251, ISSN: 2550 - 682X 
Análisis de conglomerados para la creación de un servicio: una aplicación en la hotelería

\begin{tabular}{|c|c|c|c|c|c|c|c|c|}
\hline & & & Exigentes & $-0,72349 *$ & 0,22847 & 0,006 & $-1,2763$ & $-0,1707$ \\
\hline & & \multirow{2}{*}{ Emocionales } & Prácticos & 0,10811 & 0,21051 & 0,939 & $-0,4032$ & 0,6194 \\
\hline & & & Exigentes & $-0.61538 *$ & 023115 & 0.026 & -11753 & -00555 \\
\hline & & & & & & & & \\
\hline & & \multirow{2}{*}{ Exigentes } & Prácticos & $0,72349^{*}$ & 0,22847 & 0,006 & 0,1707 & 1,2763 \\
\hline & & & Emocionales & $0.61538^{*}$ & 0.23115 & 0.026 & 0.0555 & 1.1753 \\
\hline & & & & & & & & \\
\hline & \multirow{7}{*}{$\begin{array}{l}\text { Games- } \\
\text { Howell }\end{array}$} & \multirow{2}{*}{ Prácticos } & Emocionales & $-0,10811$ & 0,22863 & 0,884 & $-0,6557$ & 0,4395 \\
\hline & & & Exigentes & $-0,72349^{*}$ & 0,19829 & 0,002 & -1.1998 & -0.2471 \\
\hline & & & & & & 0000 & & \\
\hline & & \multirow{2}{*}{ Emocionales } & Prácticos & 0,10811 & 0,22863 & 0,884 & $-0,4395$ & 0,6557 \\
\hline & & & Exigentes & $-0,61538^{*}$ & 0,21022 & 0,013 & $-1,1211$ & $-0,1097$ \\
\hline & & \multirow{2}{*}{ Exigentes } & Prácticos & $0,72349 *$ & 0,19829 & 0,002 & 0,2471 & 1,1998 \\
\hline & & & Emocionales & $0,61538^{*}$ & 0,21022 & 0,013 & 0,1097 & 1,1211 \\
\hline \multirow{13}{*}{ Servicio Imp. } & \multirow{6}{*}{ Gabriel } & \multirow{2}{*}{ Prácticos } & Emocionales & $-0,32664$ & 0,17502 & 0,182 & $-0,7517$ & 0,0984 \\
\hline & & & Exigentes & $-0,62994 *$ & 0,18995 & 0,004 & $-1,0896$ & $-0,1703$ \\
\hline & & & Prácticos & 0,32664 & 0,17502 & 0,182 & $-0,0984$ & 0,7517 \\
\hline & & Elmocionales & Exigentes & $-0,30330$ & 0,19218 & 0,309 & $-0,7688$ & 0,1622 \\
\hline & & & Prácticos & $0,62994^{*}$ & 0,18995 & 0,004 & 0,1703 & 1,0896 \\
\hline & & & Emocionales & 0,30330 & 0,19218 & 0,309 & $-0,1622$ & 0,7688 \\
\hline & & & Emocionales & $-0,32664$ & 0,19465 & 0,222 & $-0,7944$ & 0,1412 \\
\hline & & Prácticos & Exigentes & $-0,62994^{*}$ & 0,18010 & 0,003 & $-1,0654$ & $-0,1945$ \\
\hline & & & Prácticos & 0,32664 & 0,19465 & 0,222 & $-0,1412$ & 0,7944 \\
\hline & How11 & Emocionales & & & & & & \\
\hline & & & Exigentes & $-0,30330^{*}$ & 0,12597 & 0,050 & $-0,6064$ & $-0,0002$ \\
\hline & & & Prácticos & $0,62994^{*}$ & 0,18010 & 0,003 & 0,1945 & 1,0654 \\
\hline & & & Emocionales & $0,30330^{*}$ & 0,12597 & 0,050 & 0,0002 & 0,6064 \\
\hline & & & Emocionales & $-0,26255$ & 0,23360 & 0,598 & $-0,8299$ & 0,3048 \\
\hline Socio Imp. & Gabriel & Practicos & Exigentes & $-2,21310^{*}$ & 0,25353 & 0,000 & $-2,8266$ & $-1,5996$ \\
\hline & & Emocionales & Prácticos & 0,26255 & 0,23360 & 0,598 & $-0,3048$ & 0,8299 \\
\hline
\end{tabular}


María Claudia Rivera Prado, Arturo Damián Rodríguez Zambrano, Sandy Lisbeth Hormaza Villafuerte

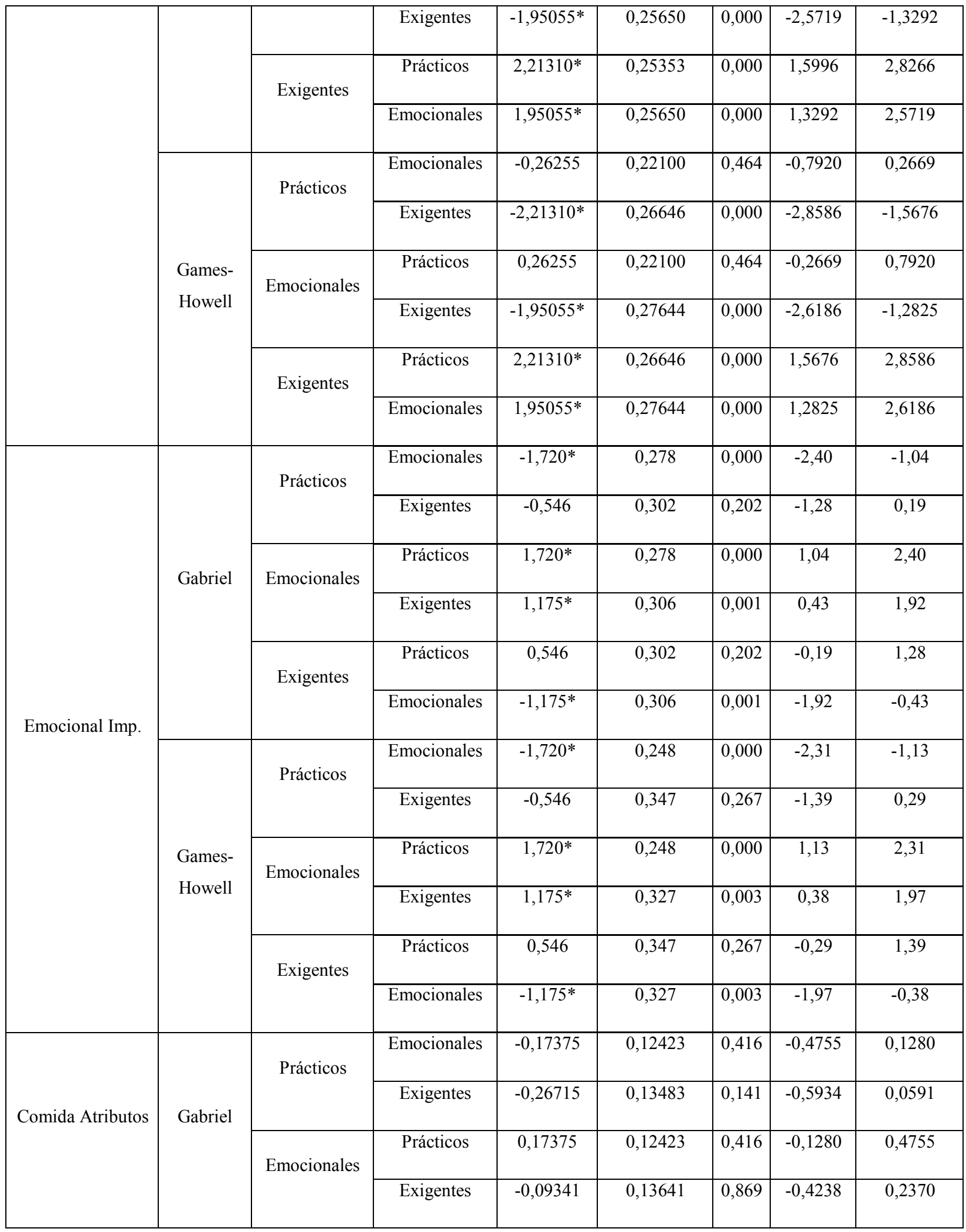

\section{6}

Pol. Con. (Edición núm. 29) Vol. 4, No 1, enero 2019, pp. 223-251, ISSN: 2550 - 682X 
Análisis de conglomerados para la creación de un servicio: una aplicación en la hotelería

\begin{tabular}{|c|c|c|c|c|c|c|c|c|}
\hline & & & Prácticos & 0,26715 & 0,13483 & 0,141 & $-0,0591$ & 0,5934 \\
\hline & & & Emocionales & 0,09341 & 0,13641 & 0,869 & $-0,2370$ & 0,4238 \\
\hline & & & Emocionales & $-0,17375$ & 0,12611 & 0,358 & $-0,4760$ & 0,1285 \\
\hline & & & Exigentes & $-0,26715$ & 0,13853 & 0,140 & $-0,6001$ & 0,0658 \\
\hline & Games & & Prácticos & 0,17375 & 0,12611 & 0,358 & $-0,1285$ & 0,4760 \\
\hline & Howell & & Exigentes & $-0,09341$ & 0,12366 & 0,732 & $-0,3918$ & 0,2050 \\
\hline & & & Prácticos & 0,26715 & 0,13853 & 0,140 & $-0,0658$ & 0,6001 \\
\hline & & & Emocionales & 0,09341 & 0,12366 & 0,732 & $-0,2050$ & 0,3918 \\
\hline & & & Emocionales & $-0,30502$ & 0,19038 & 0,299 & $-0,7674$ & 0,1574 \\
\hline & & & Exigentes & $-0,93139^{*}$ & 0,20662 & 0,000 & $-1,4313$ & $-0,4315$ \\
\hline & & & Prácticos & 0,30502 & 0,19038 & 0,299 & $-0,1574$ & 0,7674 \\
\hline & & & Exigentes & $-0,62637^{*}$ & 0,20904 & 0,010 & $-1,1327$ & $-0,1200$ \\
\hline & & & Prácticos & $0,93139 *$ & 0,20662 & 0,000 & 0,4315 & 1,4313 \\
\hline & & & Emocionales & $0,62637^{*}$ & 0,20904 & 0,010 & 0,1200 & 10,1327 \\
\hline & & & Emocionales & $-0,30502$ & 0,20948 & 0,318 & $-0,8066$ & 0,1966 \\
\hline & & & Exigentes & $-0,93139 *$ & 0,17880 & 0,000 & $-1,3613$ & $-0,5015$ \\
\hline & Games- & & Prácticos & 0,30502 & 0,20948 & 0,318 & $-0,1966$ & 0,8066 \\
\hline & Howell & & Exigentes & $-0,62637 *$ & 0,17963 & 0,003 & $-1,0588$ & $-0,1940$ \\
\hline & & & Prácticos & $0,93139 *$ & 0,17880 & 0,000 & 0,5015 & 1,3613 \\
\hline & & & Emocionales & $0,62637^{*}$ & 0,17963 & 0,003 & 0,1940 & 1,0588 \\
\hline & & & Emocionales & $-0,58919^{*}$ & 0,18966 & 0,007 & $-1,0498$ & $-0,1286$ \\
\hline & & & Exigentes & $-1,03534^{*}$ & 0,20583 & 0,000 & $-1,5334$ & $-0,5373$ \\
\hline & & & Prácticos & $0,58919^{*}$ & 0,18966 & 0,007 & 0,1286 & 1,0498 \\
\hline IVIUSicos Atrioutos & Jaoriel & Limucionaies & Exigentes & $-0,44615$ & 0,20825 & 0,099 & $-0,9506$ & 0,0583 \\
\hline & & & Prácticos & $1,03534^{*}$ & 0,20583 & 0,000 & 0,5373 & 1,5334 \\
\hline & & LAng & Emocionales & 0,44615 & 0,20825 & 0,099 & $-0,0583$ & 0,9506 \\
\hline
\end{tabular}


María Claudia Rivera Prado, Arturo Damián Rodríguez Zambrano, Sandy Lisbeth Hormaza Villafuerte

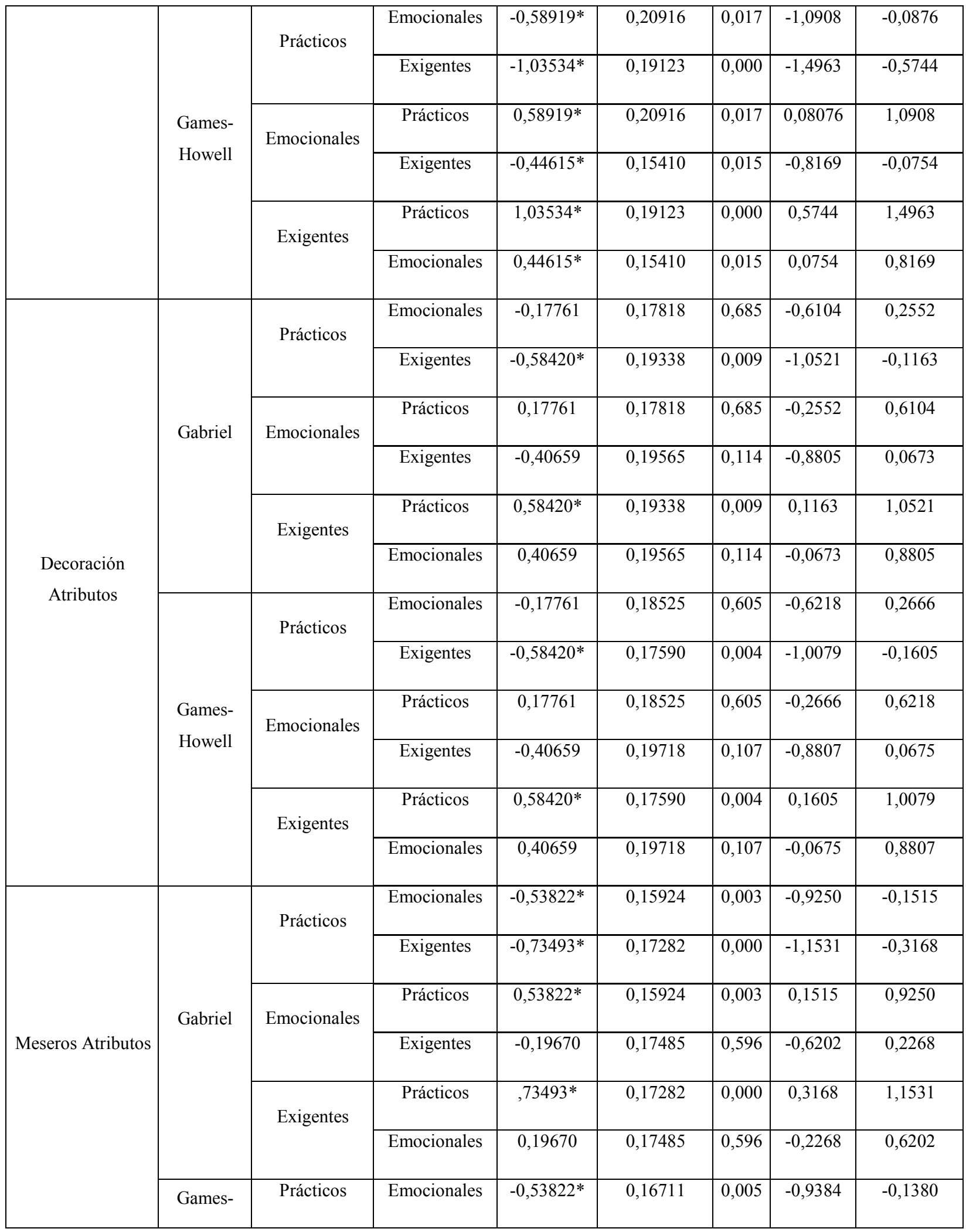

\section{8}

Pol. Con. (Edición núm. 29) Vol. 4, No 1, enero 2019, pp. 223-251, ISSN: 2550 - 682X 
Análisis de conglomerados para la creación de un servicio: una aplicación en la hotelería

\begin{tabular}{|c|c|c|c|c|c|c|c|c|}
\hline & Howell & & Exigentes & $-0,73493 *$ & 0,16647 & 0,000 & $-1,1349$ & $-0,3350$ \\
\hline & & & Prácticos & $0,53822 *$ & 0,16711 & 0,005 & 0,1380 & 0,9384 \\
\hline & & & Fxioentes & -0.19670 & 015643 & 0.425 & -0.5730 & 01796 \\
\hline & & & & & & & & \\
\hline & & & Prácticos & ,73493* & 0,16647 & 0,000 & 0,3350 & 1,1349 \\
\hline & & & Emocionales & 0,19670 & 0,15643 & 0,425 & $-0,1796$ & 0,5730 \\
\hline & & & Emocionales & $-0,29961$ & 0,15211 & 0,147 & $-0,6691$ & 0,0698 \\
\hline & & & Exigentes & $-0,56445^{*}$ & 0,16509 & 0,003 & $-0,9639$ & $-0,1650$ \\
\hline & & & Prácticos & 0,29961 & 0,15211 & 0,147 & $-0,0698$ & 0,6691 \\
\hline & & & Exigentes & $-0,26484$ & 0,16702 & 0,305 & $-0,6694$ & 0,1397 \\
\hline & & & Prácticos & $0,56445^{*}$ & 0,16509 & 0,003 & 0,1650 & 0,9639 \\
\hline & & & Emocionales & 0,26484 & 0,16702 & 0,305 & $-0,1397$ & 0,6694 \\
\hline 100 & & & Emocionales & $-0,29961$ & 0,16212 & 0,162 & $-0,6880$ & 0,0888 \\
\hline & & Prácticos & & & & & & \\
\hline & & & Exigentes & $-0,56445^{*}$ & 0,15784 & 0,002 & $-0,9437$ & $-0,1852$ \\
\hline & Games- & & Prácticos & 0,29961 & 0,16212 & 0,162 & $-0,0888$ & 0,6880 \\
\hline & Howell & - & Exigentes & $-0,26484$ & 0,14125 & 0,155 & $-0,6045$ & 0,0748 \\
\hline & & & Prácticos & $0,56445^{*}$ & 0,15784 & 0,002 & 0,1852 & 0,9437 \\
\hline & & & Emocionales & 0,26484 & 0,14125 & 0,155 & $-0,0748$ & 0,6045 \\
\hline & & & Emocionales & $-0,12819$ & 0,18335 & 0,863 & $-0,5735$ & 0,3171 \\
\hline & & & Exigentes & $-0,29522$ & 0,19900 & 0,361 & $-0,7767$ & 0,1863 \\
\hline & & & Prácticos & 0,12819 & 0,18335 & 0,863 & $-0,3171$ & 0,5735 \\
\hline & & 21 & Exigentes & $-0,16703$ & 0,20133 & ,790 & $-0,6547$ & 0,3207 \\
\hline Clima Atributos & & & Prácticos & 0,29522 & 0,19900 & 0,361 & $-0,1863$ & 0,7767 \\
\hline & & & Emocionales & 0,16703 & 0,20133 & ,790 & $-0,3207$ & 0,6547 \\
\hline & & & Emocionales & $-0,12819$ & 0,17678 &, 750 & $-00,5520$ & 0,2956 \\
\hline & Games- & Practicos & Exigentes & -029522 & 020254 & 0,321 & -0.7858 & 0.1954 \\
\hline & Howell & & & & & & & \\
\hline & & Emocionales & Prácticos & 0,12819 & 0,17678 & ,750 & $-0,2956$ & 0,5520 \\
\hline
\end{tabular}


María Claudia Rivera Prado, Arturo Damián Rodríguez Zambrano, Sandy Lisbeth Hormaza Villafuerte

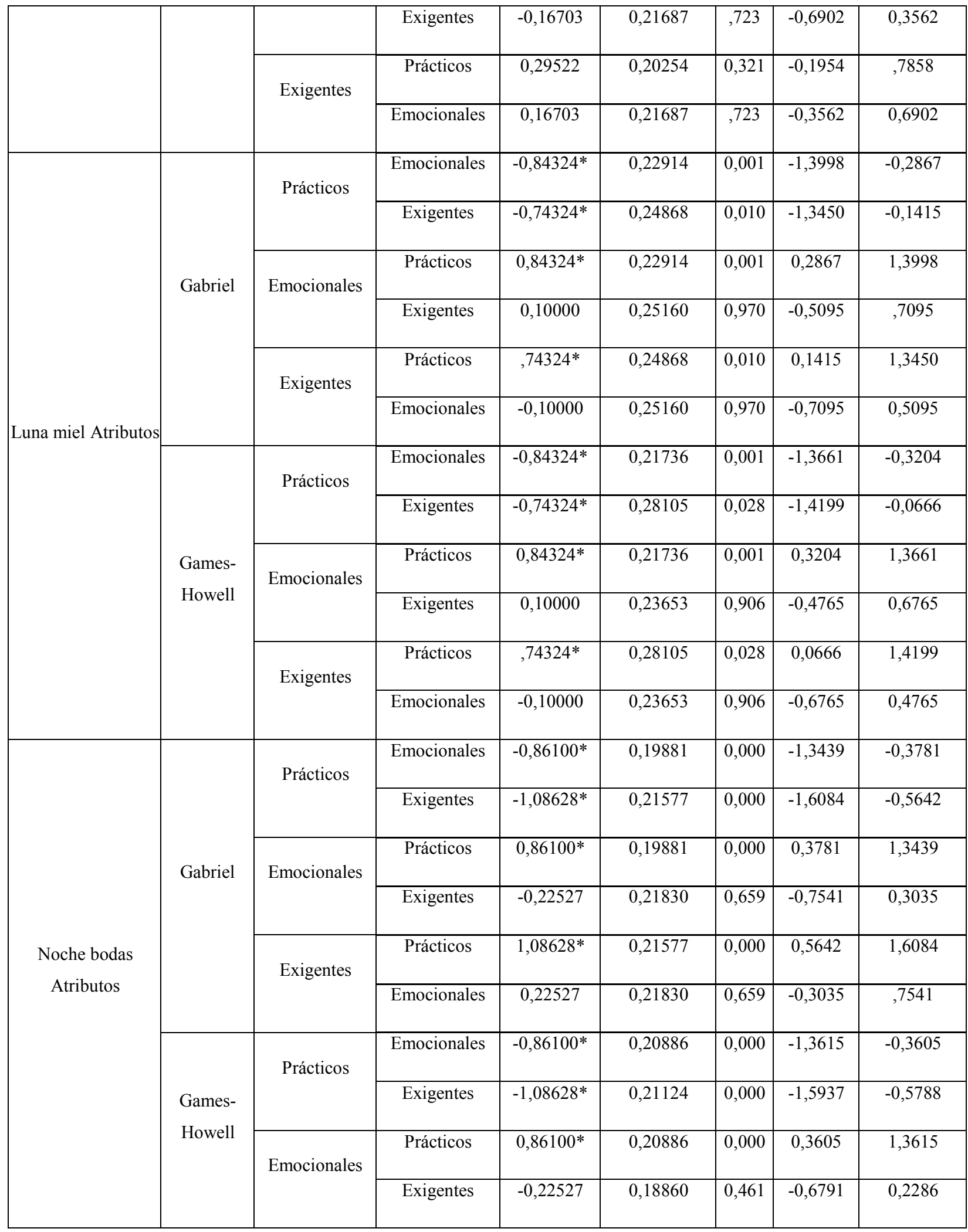


Análisis de conglomerados para la creación de un servicio: una aplicación en la hotelería

\begin{tabular}{|l|l|l|l|l|l|l|l|c|}
\hline & \multirow{2}{*}{} & \multirow{2}{*}{ Exigentes } & Prácticos & $1,08628^{*}$ & 0,21124 & 0,000 & 0,5788 & 1,5937 \\
\cline { 4 - 8 } & & & & & & & & 0,6791 \\
\hline
\end{tabular}

*. The mean difference is significant at the 0.05 level. 